Rapid Reviews COVID-19

\title{
Review 1: "Molecular Docking Studies of Aromatherapy Oils Against SARS-COV-2"
}

Kalirajan Rajagopal ${ }^{1}$

${ }^{1}$ Assistant Professor, JSS College of Pharmacy, Tamil Nadu, India

Published on: Nov 23, 2020

DOI: 10.1162/2e3983f5.b293af9b

License: Creative Commons Attribution 4.0 International License (CC-BY 4.0). 


\section{$\underline{\text { RR:C19 Evidence Scale rating by reviewer: }}$}

- Potentially informative. The main claims made are not strongly justified by the methods and data, but may yield some insight. The results and conclusions of the study may resemble those from the hypothetical ideal study, but there is substantial room for doubt. Decision-makers should consider this evidence only with a thorough understanding of its weaknesses, alongside other evidence and theory. Decisionmakers should not consider this actionable, unless the weaknesses are clearly understood and there is other theory and evidence to further support it.

$* * * * * * * * * * * * * * * * * * * * * * * * * * * * * * * * * * * * * * *$

\section{Review:}

1. The Authors used the .pdb such as 2AJF, 3SCI and 6ACK which are obtained in the year 2005, 2002, and 2018 respectively. But SARS CoV-2 was identified only in the end of the year 2019. How are the above proteins responsible for SARS CoV-2?

2. In page no.4, Section $C$, the authors state that "Each of the seven aromatherapy compounds were docked to the nine prepared receptors using AutoDock Vina." What are the nine receptors? Explain.

3. Why did you compare with camostat for docking study and compare with Remdesivir for ADME studies. Why did you not use the same standard?

4. When compared with camostat, you did not list any significant docking score. What is the significance for this study?

5. The references are not given in the uniform style. Rewrite the references in the same format as per journal's requirement. 